\title{
Complicações relacionadas ao tratamento do hipertireoidismo com radioiodo 131
}

\author{
Complications related to the treatment of hyperthyroidism with radioiodine 131 \\ Complicaciones relacionadas con el tratamiento del hipertiroidismo con yodo \\ radiactivo 131
}

\begin{abstract}
Andreza Alves Pereira ${ }^{1 *}$, Andreza Maria Pereira Alves², Andrezza Mendes Franco ${ }^{3}$, Dâmaris Eduarda de Sousa Prado ${ }^{4}$, Isadora Medrado Goulart ${ }^{5}$, Maria Julia Tribulato Silva ${ }^{6}$, Robbson Haugusto Dambros ${ }^{7}$, Tatiely Rodrigues Martins ${ }^{8}$, Yves Henrique Faria Dias ${ }^{9}$, Gustavo Francklin Milward de Azevedo ${ }^{10}$.
\end{abstract}

\section{RESUMO}

Objetivo: Evidenciar as possíveis complicações do tratamento do hipertireoidismo com radioiodo 131. Revisão bibliográfica: $O$ hipertireoidismo é uma síndrome clínica resultante da exposição dos tecidos corporais a altas concentrações de hormônios tireoidianos. As etiologias mais comuns são a Doença de Graves, adenomas hiperfuncionantes únicos, bócio multinodular tóxico e tireoidite de Hashimoto. Além disso, acomete cerca de $0,8 \%$ da população na Europa e 1,3\% nos EUA, sendo mais prevalente entre o sexo feminino e pessoas brancas. O radioiodo 131 é a primeira linha de tratamento para o hipertireoidismo, sendo considerado seguro, de fácil manuseio, baixo custo e eficiente para a terapêutica de patologias benignas e malignas da tireoide. Considerações finais: Embora essa terapia disponha diversos benefícios, foi identificado algumas complicações, como o desenvolvimento de hipotireoidismo, alterações hematológicas, pulmonares, aparecimento de tumores, cardiomiopatias, dano na tireoide de fetos, além de risco aumentado de exacerbação da oftalmopatia. Desse modo, é imprescindível que o profissional tenha atenção redobrada aos pacientes com contraindicações e avalie o risco-benefício individualmente para que o tratamento ideal seja escolhido.

Palavras-chave: Hipertireoidismo, 3-iodobenzilguanidina, Terapêutica.

\footnotetext{
ABSTRACT

Objective: To highlight the possible complications of the treatment of hyperthyroidism with radioiodine 131 . Bibliographic review: Hyperthyroidism is a clinical syndrome resulting from exposure of body tissues to high concentrations of thyroid hormones. The most common etiologies are Graves' disease, single hyperfunctioning adenomas, toxic multinodular goiter and Hashimoto's thyroiditis. In addition, it affects about $0.8 \%$ of the population in Europe and $1.3 \%$ in the USA, being more prevalent among females and white people. Radioiodo 131 is the first line of treatment for hyperthyroidism, being considered safe, easy to handle, low cost and

1 Universidade Federal do Vale do São Francisco (UNIVASF), Paulo Afonso - BA.

*E-mail: dezaalvesp@gmail.com

2 Centro Universitário IMEPAC, Araguari - MG.

${ }^{3}$ Universidade Federal do Amazonas (UFAM), Manaus - AM.

${ }^{4}$ Universidade José do Rosário Vellano (UNIFENAS), Belo Horizonte - MG.

${ }^{5}$ Universidade Brasil (UB), Fernandópolis - SP.

${ }^{6}$ Universidade Anhembi Morumbi (UAM), São Paulo - SP.

${ }^{7}$ Centro Universitário de Pato Branco (UNIDEP), Pato Branco - PR.

${ }^{8}$ Universidade Federal de Roraima (UFRR). Boa Vista - RR.

${ }^{9}$ Faculdade de Ciências Médicas e da Saúde de Juiz de Fora (FCMS/JF), Juiz de Fora - MG.

10 Escola de Medicina da Santa de Misericórdia, Vitória - ES.
} 
efficient for the treatment of benign and malignant thyroid pathologies. Final considerations: Although this therapy has several benefits, some complications have been identified, such as the development of hypothyroidism, hematological and pulmonary changes, appearance of tumors, cardiomyopathies, damage to the thyroid of fetuses, and increased risk of exacerbation of ophthalmopathy. Thus, it is essential that the professional pay extra attention to patients with contraindications and assess the risk-benefit individually so that the ideal treatment is chosen.

Keywords: Hyperthyroidism, 3-lodobenzylguanidine, Therapeutics.

\section{RESUMEN}

Objetivo: Destacar las posibles complicaciones del tratamiento del hipertiroidismo con radioyodo 131. Revisión bibliográfica: El hipertiroidismo es un síndrome clínico resultante de la exposición de los tejidos corporales a altas concentraciones de hormonas tiroideas. Las etiologías más comunes son la enfermedad de Graves, los adenomas únicos hiperfuncionantes, el bocio multinodular tóxico y la tiroiditis de Hashimoto. Además, afecta a cerca del $0,8 \%$ de la población en Europa y al 1,3\% en EE.UU., siendo más frecuente entre las mujeres y las personas de raza blanca. El radioyodo 131 es la primera línea de tratamiento para el hipertiroidismo y se considera seguro, fácil de manejar, de bajo coste y eficaz para el tratamiento de patologías tiroideas benignas y malignas. Consideraciones finales: Aunque esta terapia tiene varios beneficios, se han identificado algunas complicaciones, como el desarrollo de hipotiroidismo, cambios hematológicos y pulmonares, aparición de tumores, cardiomiopatías, daños en la tiroides de los fetos y aumento del riesgo de exacerbación de la oftalmopatía. Por ello, es fundamental que el profesional preste especial atención a los pacientes con contraindicaciones y evalúe el riesgo-beneficio de forma individual para que se elija el tratamiento ideal.

Palabras clave: Hipertiroidismo, 3-Yodobencilguanidina, Terapéutica.

\section{INTRODUÇÃO}

O hipertireoidismo é uma condição clínica na qual há hormônios tireoidianos, triiodotironina (T3) e tiroxina (T4), em excesso no organismo (KRAVETZ I, 2016). Tal disfunção tireoidiana é relativamente comum, em especial no sexo feminino. A prevalência dessa tireotoxicose é de $0,8 \%$ na Europa e 1,3\% nos EUA, aumenta quanto maior a idade e é evidenciada com maior frequência em pessoas brancas quando comparada a outras etnias. A incidência de hipertireoidismo leve também se apresenta mais elevada em áreas com deficiência de iodo (DE LEO S, et al., 2016).

Entre as causas, destacam-se como as mais comuns o hipertireoidismo autoimune, também chamado de bócio tóxico difuso ou Doença de Graves (DG), adenomas hiperfuncionantes únicos e bócio multinodular tóxico. Além destas, existem também como causas a tireoidite de Hashimoto, De Quervain, silenciosa, pósparto e tireoidite causada por medicamentos (CORRALES-HERNÁNDEZ JJ, et al., 2019).

O tratamento para essa condição inclui medicamentos antitireoidianos, radioiodo 131 e/ou cirurgia a depender da etiologia da doença (KITAHARA CM, et al., 2020; DE LEO S, et al., 2016) com o intuito de diminuir a quantidade de hormônio produzidos e/ou alterando esse fato indiretamente pela retirada do volume da glândula tireóide (TAMAYO-ALONSO P, et al., 2019).

Nos EUA, a radioiodoterapia é considerada como tratamento de primeira linha, enquanto no Brasil, os antitireoidianos são mais utilizados, apesar de não estarem relacionados a moderadas e altas taxas de cura. Independente das complicações que serão aqui relatadas, a terapia com radioiodo 131 é em geral, de baixo custo, além de ser empregada com sucesso no diagnóstico e terapêutica de doenças benignas e malignas da tireoide (DE LEO S, et al., 2016).

A terapia com radioiodo vem sendo utilizada por quase 80 anos e, desde então, informações sobre sua eficácia e seus efeitos adversos têm sido abordadas. Entre as vantagens dessa radioterapia estão a cura 
eficiente para o hipertireoidismo, a fácil administração e a não necessidade de internação hospitalar. Entretanto, quanto às desvantagens, estão o provável aparecimento de hipotireoidismo, que se apresenta em 5 a $50 \%$ dos casos no primeiro ano após o tratamento, a vagarosa indução do eutireoidismo, o possível agravo da oftalmopatia, a necessidade de adiar a gestação por até 6 meses após a administração e de cumprir as indicações de proteção radiológica, além da possibilidade de desenvolver arritmia caso ocorra tireoidite radioativa aguda no caso de idosos ou de doença cardíaca estabelecida (TAMAYO-ALONSO P, et al., 2019).

Diante do exposto, o objetivo do presente estudo foi realizado uma revisão crítica da literatura, a fim de evidenciar as possíveis complicações relacionadas ao tratamento do hipertireoidismo com radioiodo 131, haja vista que este é o tratamento que vem sendo preconizado na maioria das situações.

\section{REVISÃO BIBLIOGRÁFICA}

\section{Mecanismo de ação da terapia com radioiodo $\left({ }^{131}\right)$}

O tratamento do hipertireoidismo baseado na administração de doses de iodo radioativo tem por finalidade a ablação da tireóide, de modo a provocar redução dos níveis séricos de hormônios tireoidianos (diminuição da função tireoidiana), além de uma redução do volume da glândula tireoide (RACARU LV, et al., 2017), desse modo podendo ser uma modalidade de tratamento definitivo, assim como a cirurgia de retirada da tireóide, chamada tireoidectomia (GIESECKE P, et al., 2017). Outros resultados observados com o uso dessa opção terapêutica são a restauração do estado eutireoidiano, ou mais frequentemente a evolução para um quadro de hipotireoidismo pós-radioiodoterapia dentro de dois a seis meses (KRAVETS I, 2016).

O mecanismo de ação propriamente dito envolve a absorção seletiva do $\mathrm{I}^{131}$ pelo tecido tireoidiano e incorporação deste nos hormônios produzidos pela glândula (FANNING E e WARRICK JI, et al., 2018). Uma vez incorporado, o isótopo radioativo começa a liberar partículas radioativas, especialmente do tipo beta, que irão atuar nas células foliculares da tireoide e induzir a destruição gradual da glândula (BURCH HB e COOPER DS, 2015).

De maneira geral, os efeitos da radiação ionizante que determinam as propriedades ablativas do iodo radioativo incluem: danos ao material genético das células tireoidianas (uma vez que as partículas emitidas promovem a quebra das ligações moleculares e a produção de radicais livres extremamente nocivos ao DNA) e posterior indução da apoptose, com a participação da proteína p53 (TAMAYO-ALONSO P, et al., 2019).

Além disso, fatores como tamanho da glândula, presença de nódulos tireoidianos, administração de fármacos contendo iodo, idade, sexo, tratamento prévio com drogas antitireoidianas, uso de lítio, grau de intensidade do hipertireoidismo e capacidade de captação de iodo radioativo são fatores que influenciam nos resultados desta modalidade de tratamento (HAMMOND EM e VANGU MDRHW, 2016).

Esse tratamento possui duas formas de aplicação: empírica e a calculada. A primeira, é caracterizada por uma dose padrão e é mais utilizada, pelo seu custo-benefício e facilidade de aplicação, além disso não há diferenças estatisticamente significativa para a cura quando se compara com a segunda forma, apesar de sua individualidade. No entanto, é necessário destacar que apesar da eficiência não ser prejudicada, o índice de hipotireoidismo é maior do que nas doses calculadas (RACARU LV, et al., 2017).

Vale ressaltar que esses pacientes apresentam pior qualidade de vida pela escores ThyPTO e SF-36 (TORRING O, et al., 2018). Então vale uma análise de até que ponto esse tratamento é realmente benéfico, mas também procurar formas de evitar o hipotireoidismo, como feito por De Leo $S$, et al. (2016), em seu estudo usando um pré-tratamento com drogas antitireoidianas, contudo, é necessário enfatizar que esse fato prejudicou a eficácia do tratamento.

\section{Indicações e contraindicações}

A partir da produção científica que foi base para a presente investigação, observou-se que para a terapêutica do hipertireoidismo, a radioiodoterapia é utilizada no mundo desde 1941 (BESLIC N, et al., 2017), devido a sua eficácia, baixo custo, facilidade de administração e segurança (DIAZ A, et al., 2017). Essa relação, consiste na principal abordagem para o tratamento inicial de hipertireoidismo, para a recidiva da 
doença após terapia tireostática com drogas antitireoidianas (DAT) ou cirurgia, além de ser utilizada em pacientes que apresentam efeitos adversos graves, como reação alérgica, agranulocitose e hepatotoxicidade, com a terapia tireostática (BESLIC N, et al., 2017; RACARU LV, et al., 2017). Outra indicação de uso da radioiodoterapia são para pacientes selecionados com diagnóstico confirmado de câncer na tireoide bem diferenciado (papilífero ou folicular), sendo, geralmente, administrada após tireoidectomia total (FILETT S, et al., 2019).

A finalidade do tratamento do hipertireoidismo a partir da ablação com iodo radioativo (radioiodo 131, iodo 131, 131 l) é o restabelecimento do estado eutireoidiano ou tornar o paciente hipotireoidiano permanentemente (HAMMOND NE e VANGU MH, 2016). Nos Estados Unidos, o iodo radioativo é o recurso terapêutico de primeira linha para Doença de Graves. Já na Europa é usado como método definitivo, sendo indicado quando a doença não responde à administração dos medicamentos antitireoidianos (BESLIC N, et al., 2017; RACARU LV, et al., 2017; TAYLOR P, et al., 2018).

A radioterapia com iodo 131 é também empregada no tratamento de bócio multinodular e adenoma tóxico (AT). Sendo, no caso do bócio multinodular uma opção terapêutica utilizada de maneira isolada ou com estimulação prévia do TSH recombinante (ORELLANO P, et al., 2020). O uso da terapia com o radioiodo 131 diminui a necessidade de intervenção operatória, além de ser um método indolor e minimamente invasivo, sendo mais bem aceito pelos pacientes (BESLIC N, et al., 2017; ORELLANO P, et al., 2020). Outro fator de extrema importância a ser mencionado é o fato de não possuir riscos oncogenéticos significativos descritos na literatura (RACARU LV, et al., 2017).

A partir da análise dos artigos, as contraindicações absolutas para a terapêutica com iodo radioativo são: (I) gravidez, (II) lactação, (III) planejamento de gravidez para os próximos 6 meses e (VI) inaptidão de cumprir as instruções de segurança contra radiação (DE LEO S, et al., 2016). Em mulheres com interesse de engravidar, prefere-se a tireoidectomia e evita-se a terapia com medicamentos antitireoidianos e iodo radioativo em decorrência dos possíveis efeitos adversos no feto (SJOLIN G, et al., 2019). Foi observado que antes da administração da dose, deve ser prescrito exame para exclusão de gravidez a todas as mulheres em idade fértil.

Em relação aos pacientes com nódulos na tireoide, cujas amostras de biópsia são suspeitas ou diagnosticadas de câncer de tireoide, a iodoterapia é contraindicada, nesse caso, é recomendada a cirurgia (DE LEO S, et al., 2016). Sendo importante salientar que pacientes com Doença de Graves moderada a grave têm contraindicação relativa para 1131 devido ao risco aumentado de agravamento a orbitopatia destes (DE LEO S, et al., 2016). No caso de orbitopatia leve, a terapia com o iodo 131 deve ser acompanhada por uma terapêutica profilática com esteroides (DE LEO S, et al., 2016; FANNING E e WARRICK JI, et al., 2018). Nesse sentido, pacientes com orbitopatia de Graves inativa, mas sem fatores de risco, podem receber terapia com iodo radioativo sem corticosteroides (DE LEO S, et al., 2016).

\section{Eficácia}

A terapia de rádio iodo é uma das três opções para tratamento de hipertireoidismo, o uso desta varia para cada país, sendo utilizada como tratamento de primeira linha ou apenas em caso de recidivas após o tratamento com drogas antitireoidianas (HAMMOND EM e VANGU MDRHW, 2016). Sabe-se que essa terapia é segura e eficaz, além do seu baixo custo e facilidade de aplicação de acordo Diáz A, et al. (2019), este fato é demonstrado em vários estudos apresentando que seus benefícios são maiores do que seus riscos (KITAHARA C, et al., 2020). Um exemplo desse fato é o índice elevado de pessoas que precisam continuar com tratamento medicamentoso após "a cura", pois o hipotireoidismo é considerado um resultado aceitável, principalmente em pacientes com Doença de Graves (DG), pois esses apresentam maior probabilidade quando comparados com aqueles que apresentam adenoma tóxico e bócio multinodular tóxico (BESLIC N, et al., 2017; RACARU LV, et al., 2017).

De acordo com Fanning E e Warrick Jl (2018), 79,3\% dos pacientes nesse estudo tiveram remissão do hipertireoidismo com apenas uma dose, bem semelhante ao resultado de Racaru LV et al. (2017), que obteve $71 \%$ de sucesso para DG e para $77 \%$ para bócio multinodular tóxico, já no estudo de Orellano P, et al., (2020), 
apenas 38\% com para bócio multinodular tóxico teve o mesmo resultado no período de 6 meses. Além disso, o radioiodoterapia tem uma falha entre 10 a $50 \%$ dos casos, demonstrando uma ampla variação de resultados, isso pode ocorrer devido aos vários fatores que o procedimento pode ser influenciado, como: sexo, idade, volume da tireoide, uso prévio de medicamentos antitireoidianos e níveis basais de tiroxina livre, além da dose aplicada e da absorvida (SANTAROSA V, et al., 2015).

A utilização dessa terapia já é bem consolidada no meio médico, mas ainda há pesquisas sobre seus efeitos e o que pode ser realizado para melhorar sua aplicação e sua atuação. Um exemplo disso é o uso de Molecular Adsorbent Recirculating System (MARS) em conjunto com a radioterapia, principalmente em casos com disfunção no fígado. Este é um fator complicador para tratamento de hipertireoidismo, e no estudo de Zhang Q, et al., (2017), mostrou que a segurança do procedimento analisado aumentaria por melhorar as funções do fígado. Dessa forma, pessoas com essa disfunção somado ao hipertireoidismo poderá ter um tratamento já que elas não são elegíveis para o tratamento com medicamentos e consequentemente cirúrgicas.

Considerando que a radiação causa complicações e ainda não se sabe a dose ideal, e que apenas parte desta é captada pela glândula, variando entre 15 a 25\% durante 24 horas estudos pensaram formas para ampliar a absorção (KRAVETS I, 2016). E estes partiram do pressuposto que o iodo não radioativo impede a captação do I131, logo deveria ser consumido pouca quantidade de iodo não radioativo (KRAVETS I, 2016; SANTAROSA V, et al., 2015). Nesse sentido, a tentativa de uma dieta com baixo teor de iodo pré-aplicação foi testada, mas não teve alteração significativa na taxa de curados, mas notou-se uma redução do iodo excretado na urina, aumentando assim a eficácia do procedimento (SANTAROSA V, et al., 2015).

Outro estudo constatou um fator que pode aumentar a eficácia da radioiodoterapia diminuindo a necessidade de repetir o tratamento, usando lítio, pois esse inibe a liberação de hormônios da tireoide, que em excesso causam o hipertireoidismo, além de outros efeitos que promovem a diminuição do iodo livre e consequentemente maior absorção do iodo radioativo pela glândula, sendo considerado então um potencializador se usado antes da terapia (HAMMOND EM e VANGU MDRHW, 2016).

Apesar de ainda não existir um conhecimento exato da dose a ser usada, de todos os efeitos e de como potencializar os resultados, essa terapia é uma ótima opção para o tratamento de hipertireoidismo. Isso porque ela é definitiva, indolor e diminui o número de tratamentos usando a operação cirúrgica (DE LEO S, et al., 2016; BESLIC N, et al., 2017).

\section{Complicações}

O ser humano sofre influência em vários âmbitos, e isso pode alterar o funcionamento da terapia indicada, assim como o da doença e sua incidência, uma prova disso é que a maior incidência de hipertireoidismo se encontra em regiões com maior deficiência de iodo, e fumar é aparentemente um fator protetor nessas regiões, reduzindo em $40 \%$ a probabilidade do desenvolvimento da doença. Vale ressaltar, então, que a doença em questão é multifatorial, sendo difícil indicar o fator que alterou o funcionamento da glândula tireóide (CARDOZO LPA e CHACÓN LJR, 2016).

Embora seja uma opção terapêutica escolhida por muitos profissionais, apresenta algumas complicações. A tireoidite pela radiação, hipofunção tireoidiana permanente e o temor ao desenvolvimento de tumores (PARIDA GK, et al., 2016). Deste modo, o tratamento com radioiodo também apresenta efeitos em glândulas salivares, disfagia a longo prazo, infertilidade em homens e, embora rara, pode acontecer pneumonia e fibrose pulmonar em pacientes que recebem altas doses de lodo- 131 .

Bourcigaux N, et al. (2018), realizou um estudo prospectivo, longitudinal e multicêntrico em que avaliou a ocorrência de alterações na função testicular de homens entre 18 e 55 anos após a realização de radioiodoterapia. Utilizando parâmetros como análise hormonal, análise morfológica de espermatozoides, fragmentação do DNA e anormalidades cromossômicas, verificou-se a presença de alterações na produção hormonal por disfunção das células de Sertoli em destaque o aumento dos níveis de FSH, alteração na função das células de leydig relacionada com a um aumento da produção de LH e manutenção dos níveis de 
testosterona, na morfologia dos espermatozóides e até mesmo cromossômicas. Essas alterações se deram de maneira transitória e ocorrem em homens previamente hígidos.

Por outro lado, também foram relatados impactos da terapia ablativa com iodo na função gonadal feminina. Giusti M, et al., (2018), desenvolveu uma pesquisa visando analisar o comportamento da produção de Hormônio Anti-Mulleriano (AMH), preditor de reserva ovariana, em mulheres pré-menopausa que foram submetidas a radioiodoterapia. $O$ estudo avaliou dois grupos: grupo caso, com mulheres que fizeram uso do iodo radioativo e tireoidectomia; e grupo controle, com mulheres submetidas apenas à tireoidectomia. Como resultados, foi constatado que os níveis de AMH foram semelhantes em ambos os grupos, com risco baixo de infertilidade. Todavia, 1 em cada 2 pacientes apresentaram desregulação menstrual, exemplificado pela menor frequência de menstruações regulares e ciclos não ovulatórios no grupo caso em relação ao controle.

Clement SC, et al., (2015), por sua vez, conduziu uma revisão sistemática que averiguou os efeitos adversos da administração de $I^{131}$ a médio e longo prazo, tendo como resultados uma disfunção feminina transitória em $28 \%$ das pacientes, mas sem efeitos deletérios em longo prazo, sendo que a prevalência e a gravidade desses efeitos aumentam de forma diretamente proporcional ao aumento cumulativo da exposição ao isótopo radioativo. Além disso, destacou-se que esse tratamento não está relacionado a abortos, mas sim a um aumento no número de partos prematuros, sendo que esse último pode ser causado pelo não controle dos hormônios tireoidianos. Em quatro estudos dessa revisão foi possível afirmar que mulheres entre 35 a 59 anos tratadas com radioterapia obtiveram uma redução significativa na taxa de natalidade quando comparadas a mulheres com a mesma idade, porém não tendo passado pelo procedimento.

Além disso, pacientes também apresentam leucopenia e plaquetopenia em que podem persistir por até um ano (TAMAYO-ALONSO P, et al., 2019). Ademais, a não garantia da solução definitiva do hipertireoidismo, seu uso causa hipotireoidismo fetal e danos à glândula do bebê, apresenta mortalidade maior em pacientes que foram tratados, porém o hipertireoidismo não foi solucionado (CORRALES HERNANDEZ, et al., 2019).

O hipertireoidismo coincide com alguns casos de câncer, levando a possibilidade de interligação dessas duas ocorrências. Apesar disso, não se deve desconectar esse fato da radioiodoterapia, a qual em longo prazo, tem sido a causa de cânceres malignos em paciente que foram tratados com essa terapia e tiveram índice de morte por câncer mais alto (KITAHARA C, et al., 2020). Isso se deve ao fato que a radiação não se limita àquelas células-alvo, e portanto, alteram a estabilidade do genoma (TAMAYO-ALONSO P, et al., 2019).

Porém, estudos sobre isso são apenas observacionais, portanto, não há uma real comparação com a população no geral, apenas com aqueles com diagnóstico positivo para hipertireoidismo, e por isso a atenção aos pacientes com contraindicações deve ser redobrada para que o tratamento ideal seja escolhido (KITAHARA C, et al., 2020). Além disso, devido à instabilidade já citada pode ocorrer mutações cromossômicas em outras gerações, podendo resultar em câncer e em outras deficiências (TAMAYOALONSO P, et al., 2019).

Outro aspecto a ser mencionado, é que a Comissão Internacional de Proteção Radiológica estima que o risco de dano genético associado com exposição ao tratamento de iodo radioativo é de aproximadamente $0,005 \%$, considerando menor que o risco espontâneo de tais anormalidades. De acordo com a literatura, a possibilidade de o tratamento induzir carcinogênese, não existem evidências de haver aumento de risco de leucemia, câncer de tireoide ou de outras malignidades (CARDOZO LPA e CHACÓN LJR, 2016).

Apesar disso, a radioiodoterapia precisa entre 4 a 12 meses para permanecer em equilíbrio e o corpo retornar a homeostasia, isso provoca um estresse no sistema cardiovascular o que eleva os riscos do paciente apresentar doenças nesse sistema, e isso é demonstrado pelo aumento de hospitalizações e de mortalidade devido a alguma alteração nele. Essas alterações poderão se apresentar de diversas formas: arritmias, insuficiência cardíaca, cardiomiopatias, hipertensão (ESSI R, et al., 2017).

Esse fator corrobora com as comorbidades que surgem com o envelhecimento, as quais interferem diretamente na qualidade de vida dos pacientes, sendo afetada principalmente por sintomas físicos como cansaço e vida sexual prejudicada. Nesse sentido, é necessário sempre analisar o histórico familiar em relação às complicações de cada paciente (TORRING O, et al., 2018). 


\section{CONSIDERAÇÕES FINAIS}

Em virtude do que foi mencionado, percebe-se que a terapia com radioiodo é considerada segura em grande parte dos pacientes por ser resolutiva e evitar intervenções cirúrgicas. No entanto, essa exposição pode gerar danos severos ao organismo como alterações hematológicas, pulmonares, surgimento de neoplasias, distúrbios de condução e anormalidades na contração e relaxamento muscular das câmaras cardíacas, além de hipofunção da glândula tireóide. Da mesma forma, é preciso ter atenção quanto às contraindicações que a terapia determina, principalmente no grupo de gestantes, lactantes e mulheres em fase de planejamento da gravidez, por conta do risco teratogênico. Nesse sentido, é necessário que o profissional, ao prescrever a terapia, seja capaz de avaliar o risco-benefício de maneira individualizada para que o tratamento ideal seja escolhido.

\section{REFERÊNCIAS}

1. BESLIC N, et al. Incidence of Hypothyreoidism after Radioactive lodine-I131 Treatment in Dependance of Hyperthyreoidism Etiology and Therapy Dose. MED ARCH, 2017; 71(4): 270-273.

2. BOURCIGAUX N, et al. Impact on testicular function of a single ablative activity of $3.7 \mathrm{GBq}$ radioactive iodine for differentiated thyroid carcinoma. Human Reproduction, 2018; 33(8): 1408-1416.

3. BURCH HB, COOPER, DS. Management of graves disease a review. In JAMA - Journal of the American Medical Association. American Medical Association, 2015; 314(23): 2544-2554.

4. CARDOZO LPA, CHACÓN LJR. Factores predisponentes de la disfunción tiroidea. Medinterna, $2016 ; 32$ (2): 115-123.

5. CLEMENT SC, et al. Intermediate and long-term adverse effects of radioiodine therapy for differentiated thyroid carcinoma - a systematic review. Cancer treatment Rev, 2015; 41(10): 925-934.

6. CORRALES-HERNÁNDEZ JJ, et al. Serviço de Endocrinologia e Nutrição. Hospital das Clínicas Universitárias, 2019; 11(3): 273-281.

7. DE LEO S, et al. Hipertireoidismo. Departamento de Ciências Clínicas e Saúde Comunitária Universidade de Milão, 2016; 388(8): 906-918.

8. DIAZ AL, et al. Obtenção combinada de parâmetros para planejamento dosimétrico 2D e 3D de tratamentos com I131 em hipertireoidismo. Ciências Nucleares, 2017; 61:16-20.

9. ESSI R, et al. Morbidade e mortalidade cardiovascular após o tratamento do hipertireoidismo com iodo radioativo ou tireoidectomia. Thyroid, 2018; 28(9): 1111-1120.

10. FANNING E; WARRICK JI. Radioiodine treatment for graves' disease: a 10-year Australian cohort study. BMC Endocrine Disorders, 2018; 18(1): 94.

11. FILETTI S, et al. Thyroid cancer: ESMO Clinical Practice Guidelines for diagnosis, treatment and follow-up. 2019

12. GIESECKE P, et al. All-cause and cardiovascular mortality risk after surgery versus radioiodine treatment for hyperthyroidism. Wiley Online Library, 2017. 105(3): 279-286.

13. GIUSTI M, et al. Anti-Müllerian hormone in pre-menopausal females after ablative radioiodine treatment for differentiated thyroid cancer. Endocrine, 2018; 60(3): 516-523.

14. HAMMOND EM, VANGU MDRHW. Effect of adjuvant lithium on thyroxine (T4) concentration after radioactive iodine therapy. European Journal of Nuclear Medicine and Molecular Imaging, 2016; 43(11): 1980-1987.

15. KITAHARA C, et al. Association of Radioactive lodine, Antithyroid Drug, and Surgical Treatments With Solid Cancer Mortality in Patients With Hyperthyroidism. JAMA Network OPEN, 2020; 3(7): e209660.

16. KRAVETS I. Hyperthyroidism: Diagnosis and Treatment. In American Family Physician, 2016; 93(5): 363-370.

17. ORELLANO $P$, et al. Resultados de curto prazo do tratamento com 1131 em bócio multinodular: valor adicional da estimulação de rhTSH anterior. Rev Chil Endo Diab, 2020; 13(3): 110-117.

18. PARIDA GK, et al. Study of cytogenetic toxicity of low-dose radioiodine therapy in hyperthyroid patients using a micronuclei assay. Nucl Med Commun, 2016; 37(8): 800-4.

19. RACARU LV, et al. Clinical outcomes 1 year after empiric 131 l therapy for hyperthyroid disorders: Real life experience and predictive factors of functional response. Nuclear Medicine Communications, 2017; 38(9): 756-763.

20. SANTAROSA V, et al. Dieta pobre em iodo não melhora a eficácia do radioiodo no tratamento da doença de Graves. Arch Endocrinol Metab, 2015; 59(6): 501-506.

21. SJOLIN G, et al. The long-term outcome of treatment for Graves' Hyperthyroidism. Nat Rev Endocrinol, 2019; 29(11): $1545-1557$.

22. TAMAYO-ALONSO $P$, et al. Tratamiento con radioyodo de la patología tiroidea. Revista ORL, 2020; 11(3): 305-327

23. TAYLOR PN, et al. Global epidemiology of hyperthyroidism and hypothyroidism. Nature Reviews, endocrinology, 2018; 14: $301-316$

24. TORRING O, et al. Qualidade de vida prejudicada após terapia com radioiodo em comparação com medicamentos antitireoidianos ou tratamento cirúrgico para graves hipertireoidismo. Um acompanhamento de longo prazo com ThyPTO e SF-36. Thyroid, 2018; 29(3): 322-331.

25. ZHANG Q, et al. Combination of molecular adsorbent recirculating system and radioiodine for the treatment of concurrent hyperthyroidism and severe liver dysfunction: A restropective cohort study. Endocrine Practice, 2017; 23(2): 141-148. 